\title{
Mechanochemical pattern formation in simple models of active viscoelastic fluids and solids
}

\author{
Sergio Alonso ${ }^{1}$, Markus Radszuweit ${ }^{2,3}$, Harald Engel $^{2}$, and \\ Markus Bär ${ }^{3}$ \\ Department of Physics, Universitat Politècnica de Catalunya, Av. Dr. Marañon \\ 44-50, Barcelona, Spain \\ Technische Universität Berlin, Institute for Theoretical Physics, Hardenberstr. \\ 36, 10623 Berlin, Germany \\ Physikalisch-Technische Bundesanstalt, Abbestrasse 2-12, 10587 Berlin, \\ Germany \\ E-mail: s.alonso@upc.edu \\ October 2017
}

\begin{abstract}
The cytoskeleton of the organism Physarum polycephalum is a prominent example of a complex active viscoelastic material wherein stresses induce flows along the organism as a result of the action of molecular motors and their regulation by calcium ions. Experiments in Physarum polycephalum have revealed a reach variety of mechanochemical patterns including standing, traveling and rotating waves that arise from instabilities of spatially homogeneous states without gradients in stresses and resulting flows. Herein, we investigate simple models where an active stress induced by molecular motors is coupled to a model describing the passive viscoelastic properties of the cellular material. Specifically, two models for viscoelastic fluids (Maxwell and Jeffrey model) and two models for viscoelastic solids (Kelvin-Voigt and Standard model) are investigated. Our focus is on the analysis of the conditions that cause destabilization of spatially homogeneous states and the related onset of mechano-chemical waves and patterns. We carry out linear stability analyses and numerical simulations in one spatial dimension for different models. In general, sufficiently strong activity leads to waves and patterns. The primary instability is stationary for all active fluids considered, whereas all active solids have an oscillatory primary instability. All instabilities found are of long-wavelength nature reflecting the conservation of the total calcium concentration in the models studied.
\end{abstract}

Keywords: active cytoplasm, linear viscoelasticity, Physarum polycephalum, cell rheology, non-linear science

Submitted to: J. Phys. D: Appl. Phys. 


\section{Introduction}

The cytoplasm of the living cells is formed by filaments consisting of chains of actin protein organized in bundles by cross-linking proteins [1]. The continuous polymerization and depolymerization of filaments and the unbinding of cross-linkers may produce cytoplasmic flows at large scales of time, which permits the modeling of such flows in terms of active gel theories [2, $3,4]$. At small time scales, however, the cytoskeleton is more rigid and behaves as an elastic solid [5].

The organism Physarum polycephalum has been used as a model organism to study the filament structure of the cytoskeleton [6, 7]. In particular, the reorganization of the cytoplasm of Physarum in small protoplasmic droplets has been used to study the active properties of the cytoskeleton, because mechanical deformation waves travel through the droplet during such cellular reorganization $[8,9,10$, 11]. Other aspects of the spatiotemporal dynamics of Physarum polycephalum such as percolation of small plasmodia into a transport network [12] and the frontal extension dynamics of a bigger cell [13] have been explored experimentally recently. A current focus of (mostly) experimental and theoretical studies is the investigation of migrating plasmodia $[14,15,16,17]$.

Recently, two-phase models [18, 19, 20, 21, 22] of the cytoplasm of protoplasmic droplets of Physarum have appeared, that are composed of an active solid cytoskeleton coupled to a passive viscous cytosol. A reduction of detailed models of the cytoplasm of Physarum [19, 20, 21] gives rise to a simple and more generic model of mechanochemical pattern formation in active viscoelastic cytoskeleton [23]. Since contraction and the subsequent deformations in Physarum were correlated with low calcium concentration [9], the cited models employ an inhibitory regulator for the active stress in the cytoskeleton. It was shown, that such a model yields an oscillatory long-wavelength instability and corresponding traveling and rotating waves. Earlier on, an even simpler model for pattern formation in active fluid was introduced by Bois et al. [24], that assumes that the cytoskeleton is a simple viscous fluid, wherein active stresses increases with the concentration of molecular motors or an activating regulator. This model was found to exhibit a stationary longwavelength instability and corresponding patterns. The two latter models are at the starting point of our studies.

The second model [24] is a one-phase active fluid model of the cytoplasm that yields stationary patterns and was motivated by observations in C. Elegans cells [25]. In contrast, Radszuweit et al. [23] proposed a two-phase active poroelastic model that yields waves and was motivated by the observation of deformation waves in Physarum [8]. This model represents the cytoplasm as a two-phase material consisting of a solid sponge-like cytoskeleton and a viscous cytosolic liquid. Both cases are examples of mechano-chemical pattern formation [26, 27], i.e. they do not require chemical reaction-diffusion instabilities to structure the interior of a cell. These models assume that the passive stresses are supplemented by an active stress caused by molecular motors that depends on the concentration of the motors or on regulating agents like calcium ions [28]. It is worth mentioning that both models were extended to a situation where the regulator was reacting with other biochemical species. In other words, the simple mechanochemical models were coupled to reactiondiffusion equations, yielding patterns stemming from short-wave length Turing-type instabilities. Bois et al. showed already that the coupling of reaction-diffusion equations to a mechanochemical model for an active fluid enhances the tendency of a system for pattern forming instabilities [24]. Alonso et al. observed a similar trend for the coupling of the active poroelastic equations to reaction-diffusion equations [21].

In the remainder we will, however, disregard the effect of reaction-diffusion processes mechanochemical pattern formation. Instead emphasis is on the effect different possible models for the passive viscoelastic properties of the active cytoplasm using the simple model for an active fluid used in [24] as a starting point. As in previous work [23], we keep the simple expression for the active stress introduced there, but vary the assumptions regarding the passive material properties that enter the passive stress contribution. For simplicity, we confine our study to models based on linear elasticity. Our studies addresses both viscoelastic active fluids (described by the Maxwell resp. Jeffreys model) as well as viscoelastic active solids (described by the Kelvin-Voigt resp. Standard model). The key goal of this work is to investigate the influence of these passive stresses on pattern formation for parameters that roughly match the condition in the cytoplasm of Physarum and, in general, of living cells.

Both, active fluid and solid model for the cell, have been extensively employed in the study of the viscoelastic properties of the cytoskeleton [5] and of the rheology of different types of living cells [1]. Cell rheology $[29,30]$ is the field in which the interaction between the viscoelastic properties and the mechanical response of living cells [31] are studied. A typical assumption is that the cell material can have both solid and fluid characteristics. Although the mechanics of typical eukaryotic cells were found to be solid only at time scales shorter than 1 second [32] and to exhibit fluid characteristics at longer time scales [33], viscoelastic solid models have been 
successfully employed for reproducing measurements of viscoelastic properties of living cells [34]. The combination of a viscoelastic approach with active stress can produce pattern formation in living cells, and in particular mechanochemical waves, which can produce deformation patterns in protoplasmic droplets of Physarum polycephalum [8, 21]. More examples are also possible, for instance cortical oscillations are able to move periodically the nucleus in fibroblasts $[35,36]$ or to produce actin pattern formation in epithelial cells in Drosophila [37].

This manuscript is organized as follows: in the next section, we present the basic ingredients of a simple one-phase description of the active cytoskeleton, and discuss the inclusion of active stress and viscoelastic properties into the model. In section 3 we carry out a linear stability analysis for four different models of active viscoelastic solids (KelvinVoigt and standard model) and active viscoelastic fluids (Maxwell and Jeffrey model) and identify parameter regions where spatially homogeneous steady states are unstable indicating pattern formations. We also address the non-linear dynamics of the patterns and waves emerging in the standard and Jeffrey models by numerical simulations. In section 4 , a comparison of numerically simulated patterns in one model with linear elasticity (standard model) to a description based on nonlinear elasticity is presented. Finally, section 5 provides a short summary and we discuss the results.

\section{Model}

The actomyosin cytoskeleton has been modelled at the mesoscopic scale as an active gel [2] in particular at the cellular cortex [24], the region of the cytoskeleton close to the membrane. The actomyosin network is modelled by a single gel phase, which deforms due to the active stress of the myosin.

\subsection{Active stress}

The total stress in the cytoskeleton $\sigma$ is the sum of the active tension $T(c)$ and the viscoelastic stress $\sigma_{v e}$ of the network, i.e. $\sigma=T(c)+\sigma_{v e}$. The viscoelastic properties of the cytoskeleton characterize the response of the cell to the deformations, see Fig.(1) for a sketch, and Sec. (2.2) for more details. Neglecting inertial forces, the force balance obtained from such a total stress reads:

$\partial_{x} \sigma_{v e}+\partial_{x} T(c)-\gamma v=0$,

where $\gamma$ is the friction coefficient and describes e.g. the friction that occurs if the cytoskeleton (actin cortex) moves relative to the membrane. The dimensionless, scaled concentration, $c$, is assumed to determine the strength of the active tension. The quantity $c$ represents either the concentration of molecular motors (direct regulation of active tension), see e.g. [24], or of a biochemical species that controls the motor activity (indirect regulation of active tension), see $e . g$. [23]. Therefore, the active stress $T(c)$ and the resulting deformation of the actomyosin cytoskeleton depends on the local concentration $c$ as

$T(c)=T_{0}+\xi \frac{c}{K_{c}+c}$,

where $T_{0}$ is the active tension due to other active sources. The sign of $\xi$ can be positive or negative depending on whether the concentration $c$ activates [24] or inhibits [23] the active tension, respectively. The active tension may also be affected by the concentrations of two different competing species, as was assumed in a recent extended model for an active cellular fluid [38]. Apart from the action of motorregulating proteins, calcium ions are known to inhibit the action of myosin motors in Physarum [28], while they activate myosin in muscle cells [39].

Throughout this paper, we are considering the cell material as a single phase fluid or solid. Therefore, the velocity $(v)$ indicates the deformation $(u)$ of the cytoskeleton. Both quantities are related by the simple relation $\partial_{t} u=v$. This relation is quite similar to the incompressibility condition in two-phase models that relates the motion of the solid network of the cytoskeleton with the velocity of the cytosol. The only difference lies in the sign of the term with the active stress $T(c)$, which is negative instead of positive, for details see [23]. While an active fluid is appropriately described by the single-phase approach, an active solid could be coupled to a passive fluid in which the regulator of the active tension is transported as proposed in [23]. Such a two-phase poroelastic description leads, however, to an analogous description as the one phase model of an active solid presented here. In particular, the two-phase poroelastic model has exactly the same instabilities and dynamical behavior as the active solid model.

Biochemical macromolecules diffuse inside of the cell. The diffusion coefficient for proteins inside living cells depends on several factors, although the most relevant is their weight [40]. A characteristic value for the diffusion of a protein inside the cytoplasm is around $D=10 \mu \mathrm{m}^{2} / \mathrm{s}$. For smaller molecules, for example calcium, this coefficient is typically larger.

On the other hand, inside the cell active transport occurs due to the generation of flows of the cytoplasm. Such cytoplasmic streaming can organize and move different species coherently [41].

The concentration of such a biochemical component with passive, i.e. diffusive, and active, i.e. advection, transport follows a diffusion-advection equation. 


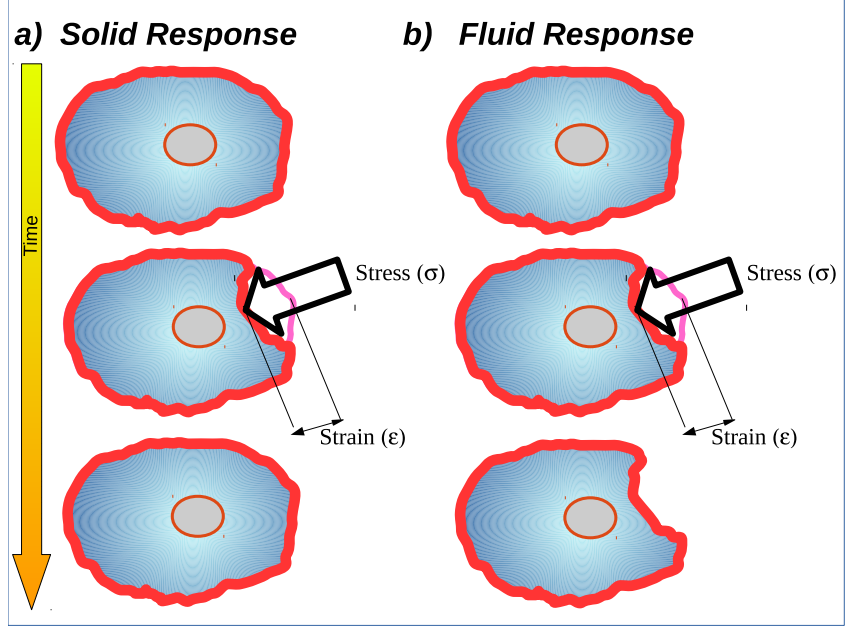

Figure 1. Comparison between an elastic (a) and a viscous (b) response of a cell to an external stress. An elastic cell recovers the initial shape when the stress is removed (a). A viscous cell adapts and does not recover the initial shape (b).

$\partial_{t} c=-\partial_{x}(v c)+D \partial_{x}^{2} c$

for the diffusion-advection equation and

$T(c)=T_{0}+\xi \frac{c}{1+c}$,

for the dependence of the active stress on the scaled concentration $c$ of the regulating species.

\subsection{Viscoelasticity}

The response of a cell to a deformation is not unique and depends on the type of the cell or on the time scale and amplitude of the perturbation. For certain types of cells this response is elastic and they behave as solids, i.e. after an initial perturbation the cell recovers the original shape, see Fig.1(a). However, under other conditions the response is viscous and the cell behaves as a fluid, i.e. the applied stress deforms the cell shape plastically and the deformation remains, see Fig.1(b). The stress $(\sigma)$ is related with the local force per unit of area and is usually given in the unit of kilopascal. The strain $(\epsilon)$ is the deformation $(u)$ per unit of length of the material. It is a non-dimensional quantity and corresponds in continuum mechanics to the spatial derivative of the deformation: $\epsilon=\partial u / \partial x$. The strain is limited therefore to $\epsilon<1$. Furthermore, the models discussed below will all be based on linear elasticity and they are typically valid for small strain $(\epsilon \ll$ 1). Traditional viscoelastic models are represented by an equivalent circuit composed of a small number of springs and dashpots that may either exhibit solid- or fluid-like elastic responses. The term $\sigma_{v e}$ in Eq.(1) is computed from these elements, see e.g. [42]. It is important to note that those springs and dashpots do not have a direct physical interpretation. Hence, the resulting models are phenomenological. For example the elastic and viscous responses shown in Fig.1(a) and (b), may be modelled in their simplest form as a spring, see Fig. 2(a), which returns the border of the cell to the original position and a dashpot, see Fig. 2(b), which deforms and adapts to the new position.
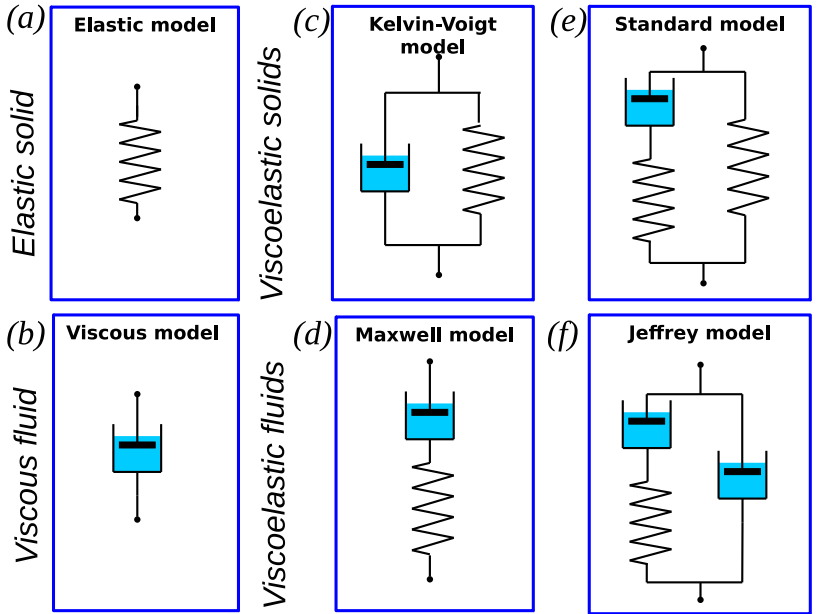

Figure 2. Different representations of the viscoelastic models in terms of springs and dashpots for viscoelastic solids and fluids.

Typically simple viscoelastic models used to describe cellular dynamics combine both types of responses (the viscous dashpot and the elastic spring). For a selection of simple viscoelastic models that we will analyse in the remainder of this paper see Fig.2. We differentiate between two basic types of models depending on the final response:

- Viscoelastic solids: after the initial stress the cell recovers its original shape. There is single equilibrium value of the strain and the spring recovers the original value after the retreat of the external stress, see examples of the stress-strain relations in Fig.3(a,c,e).

- Viscoelastic fluid: the stress produces a permanent deformation. The dashpot does not have an unique equilibrium strain, see examples of the stress-strain relations in Fig.3(b,d,f).

With the combination of springs and dashpots either in parallel of in series, we can recover a spectrum of possible responses. In Fig. 3 we show the responses of the viscoelastic models shown in Fig.2 to a constant external stress that is applied for 10 seconds. For an elastic response, the strain basically follows the applied stress and relaxes back zero when the stress disappears. For a viscous model, the strain continuously increases till the stress is removed.

The Kelvin-Voigt and the Maxwell models are the two simplest models of viscoelasticity. While the 

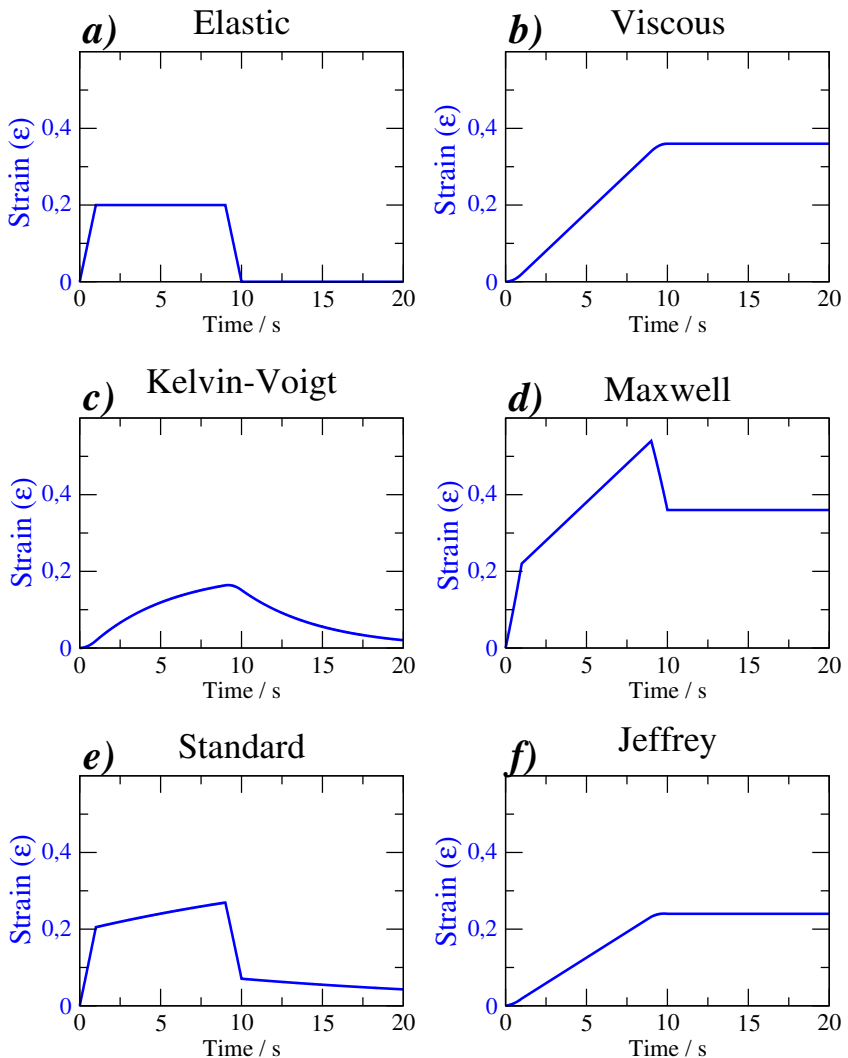

Figure 3. Comparison among the different viscoelastic models of the response, i.e. induced strain, to an external stress. The curves can be derived from expressions obtained in Appendix B Parameter values employed: $\sigma_{0}=1 k P a, E=5 \mathrm{kPa}, \eta=25 \mathrm{kPa}$ $\mathrm{s}$, and for the last cases: $E_{1}=E_{2}=E / 2$ and $\eta_{1}=\eta_{2}=\eta / 2$.

Kelvin-Voigt model represents a solid, see Fig.2, and the strain appears to saturate after a certain time to a particular value (here to $\epsilon=0.2$ at 10 seconds in Fig.3), the Maxwell model represents a fluid, see Fig.2, and the strain continuously grows for constant stress, see Fig.3. Both models has been used frequently in generic models of viscoelastic active gels.

The Standard and the Jeffrey models of viscoelasticity combine three elements, see Fig.2, to obtain more complex responses, see Fig.3. For a longer review on linear viscoelastic models we refer to [42].

\subsection{Parameter values}

Living cells are very heterogeneous and the values of mechanical parameters strongly differs even inside of the same cell at the same time [43]. Measurements of viscoelastic parameters can be done by magnetic and optical tweezers, atomic force microscopy, particle tracking and other techniques, see [29] for more detailed list and references.

Even a single interpretation of a single experiment is complicated, because it is often not easy to separate the properties of the membrane, from the ones of
Table 1. Characteristic value of the viscoelastic parameters for different living cells obtained in the bibliography.

\begin{tabular}{lll}
\hline Parameter & Cell & Value \\
\hline$E$ & melanoma cells & $1-10 \mathrm{kPa}[32]$ \\
& human platelets & $1-50 \mathrm{kPa}[44]$. \\
& fibroblasts & $1-2 \mathrm{kPa}[45]$, \\
& & $20-40 \mathrm{kPa}[34]$ \\
\hline$\eta$ & fibroblasts & $13 \mathrm{kPa} \mathrm{s}[45]$ \\
& & $2-4 \mathrm{kPa} \mathrm{s}[34]$ \\
\hline$\tau$ & fibroblasts & $0.1 \mathrm{~s}[34]$ \\
& & $40 \mathrm{~s}[45]$ \\
& drosophila oocytes & $0.5 \mathrm{~s}[46]$ \\
& endothelial cell & $10 \mathrm{~s}[47]$ \\
& C. Elegans embrio & $5 \mathrm{~s}[48]$ \\
\hline
\end{tabular}

Table 2. Generic Values of the parameters employed for the different models discussed in the manuscript.

\begin{tabular}{lll}
\hline Parameter & Value & Meaning \\
\hline$E$ & $5 \mathrm{kPa}$ & Elastic modulus \\
$\eta$ & $25 \mathrm{kPa} \mathrm{s}$ & cytoskeleton viscosity \\
$\ell$ & $10 \mu \mathrm{m}$ & Hydrodynamics length \\
$D$ & $10 \mu \mathrm{m}^{2} / \mathrm{s}$ & Diffusion coefficient \\
$\gamma$ & $0.25 \mathrm{kPa} \mathrm{s} \mu \mathrm{m}^{-2}$ & friction coefficient \\
\hline
\end{tabular}

the cytoskeleton or the cytosol with knowing only the overall viscoelastic properties of a cell measured in experiments. Estimates of concrete values are nevertheless obtained from experimental data and differ from cell to cell. As an example we give several characteristic values in Table 1. Elastic moduli of cells typically vary from $1 \mathrm{kPa}$ to almost $100 \mathrm{kPa}$. Similar variations are obtained in the measurements of the viscosity of the cytoskeleton network. The ratio of both parameters define the viscoelastic relaxation time $\tau=\eta / E$ which can vary over up to three orders of magnitude.

This large variation makes the choice of characteristic values in models difficult. Previous generic models based on active gels for the actomyosin cytoskeleton employed typical values of the elastic modulus of a few kilopascals, for example $E=1-10 \mathrm{kPa}$ [2] and viscoelastic relaxation times of $\tau=100 \mathrm{~s}$, which, by using $\tau=\eta / E$, corresponds to viscosities around $\eta=100-1000 \mathrm{kPa} \mathrm{s}[2]$. We have used direct measurements of the parameters for actomyosin cortex in onecell-stage of C. elegans embrio [48] as a first estimate to choose characteristic values for the elastic modulus and the viscosity, see Table 2; we are, nevertherless, vary systematically the values of these parameters in our study. From the similar works we have extracted an estimate for the the hydrodynamic length $\ell$ and the value of the friction coefficient, which appears in Eq.(1). The parameter values employed here, see Table 2 , are in agreement with other theoretical studies, 


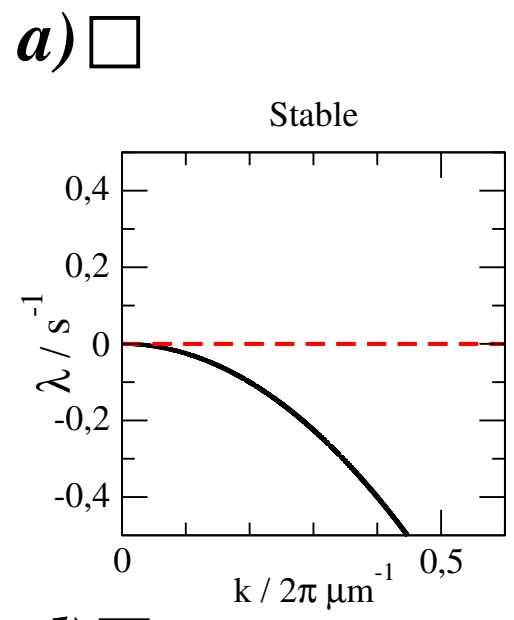

b)

c) $\square$

Unphysical (real eigenvalues) Unphysical (complex eigenvalues)

d) $\square$
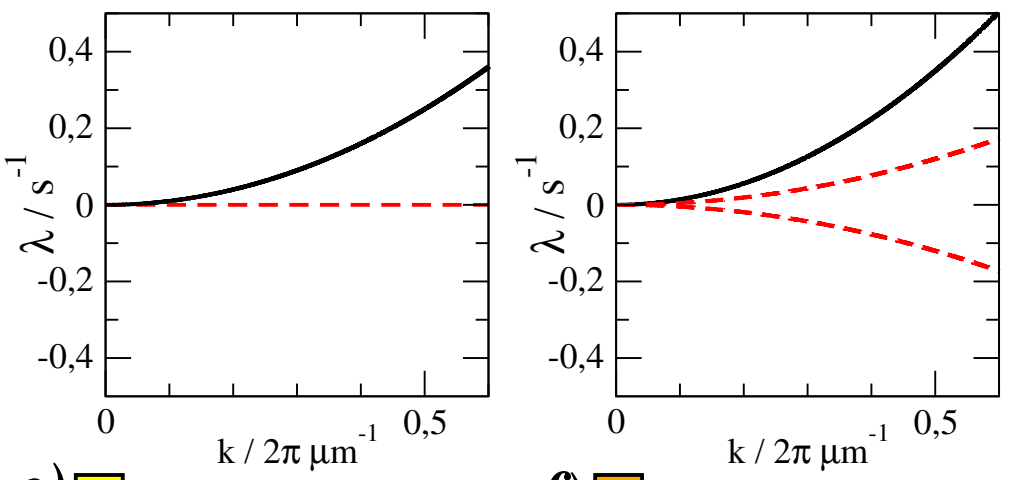

f) $\square$

Instability (real eigenvalues)

Instability (mixed eigenvalues)

Instability (complex eigenvalues)
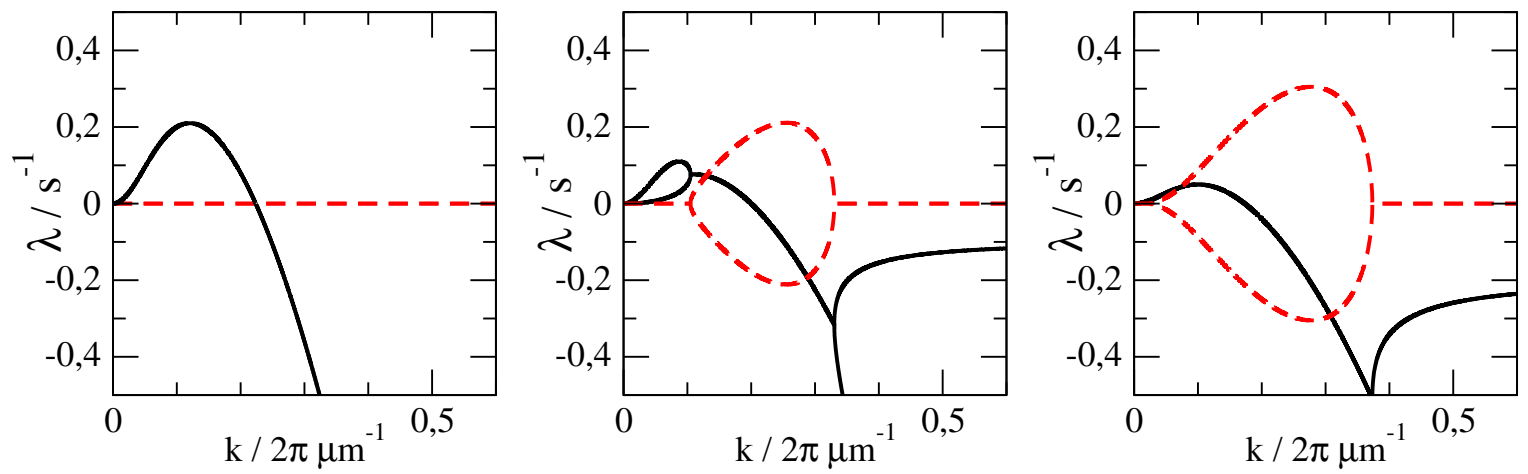

Figure 4. Dispersion relations obtained from the linear stability analysis: linearly stable homogeneous solutions, white label (a), unstable solution with all eigenvalues real and positive, blue label (b), unstable solution with all eigenvalues complex with real part positive, blue-sky label (c). Dispersion relation obtained from the linear stability analysis of eq.(16), of the viscoelastic Kelvin-Voigt model for $E=0$ (d), $M=2.5 \mathrm{kPa}(\mathrm{e})$, and $M=5 \mathrm{kPa}(\mathrm{f})$ keeping $F=6$ and $\ell=10 \mu \mathrm{m}$. Real long wave instability corresponds to a window of real unstable eigenmodes, red label $(\mathrm{d})$, complex wave instability with real leading eigenmode to a combination of real and complex unstable eigenvalues, yellow label (e), complex long wave instability to a window of complex unstable eigenmodes, orange label (f). Parameter values: $D=10 \mu \mathrm{m}^{2} / \mathrm{s}$.

for example the values $E=10 \mathrm{kPa}$, and $\eta=10 \mathrm{kPa} \mathrm{s}$ were used already early on in [49].

\section{Pattern formation in active viscoelastic models}

As a starting point for the analysis, we recall the results on one phase model of an active fluid originally proposed for the actomyosin cortex [24]. Then we introduce some classical models of linear viscoelasticity of fluids and solids. These models have been extensively applied to the modeling of the viscoelastic properties of living cells under active and passive conditions, see a collection of applications in Tab.3. First, we perform the linear stability analysis of the models and second we perform numerical simulations of the all nonlinear model for some selected cases for two more complex models of viscoelasticity.

\subsection{Linear stability analysis}

A particular homogeneous steady state is composed by the concentration $c=c_{0}$ and the velocity $v=v_{0}=0$. To study the stability of this steady state we perform a linear stability analysis, by the introduction of a spatio-temporal perturbation $c=c_{0}+\delta c e^{\lambda t+i k x}$ and $v=v_{0}+\delta v e^{\lambda t+i k x}$ and evaluate if the perturbation grows $(\lambda>0)$ or decreases $(\lambda<0)$ with time. The variable $\lambda$ is the growing rate and indicates the stability of the solutions to small perturbations. Such variable depends on the parameters of the model and on the wave number $k$, see Fig.4 for a collection of different types of dependences on $k$.

3.1.1. Starting point: Active viscous fluid The incorporation of the viscosity to the active stress in the calculation of the total stress in Eq. (1) permits the 


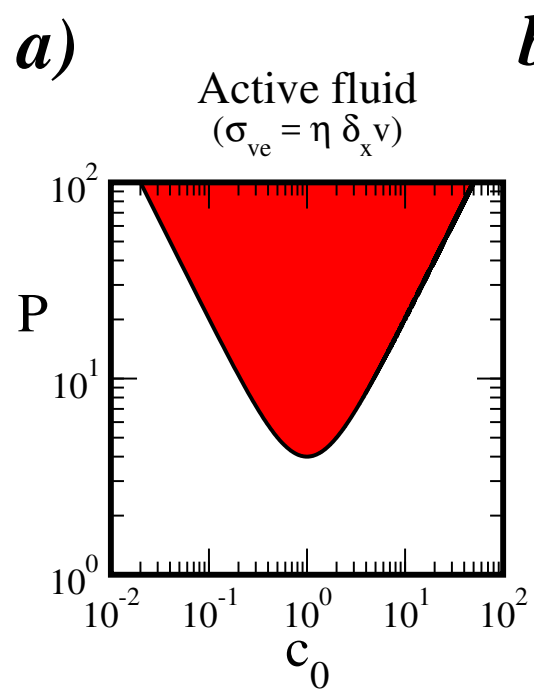

b)

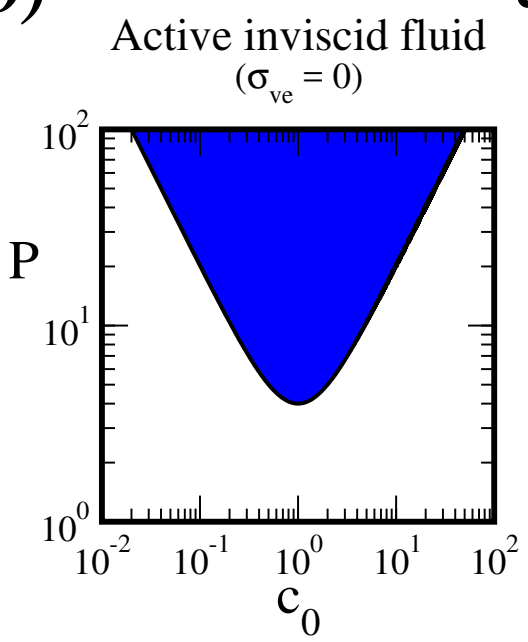

c)

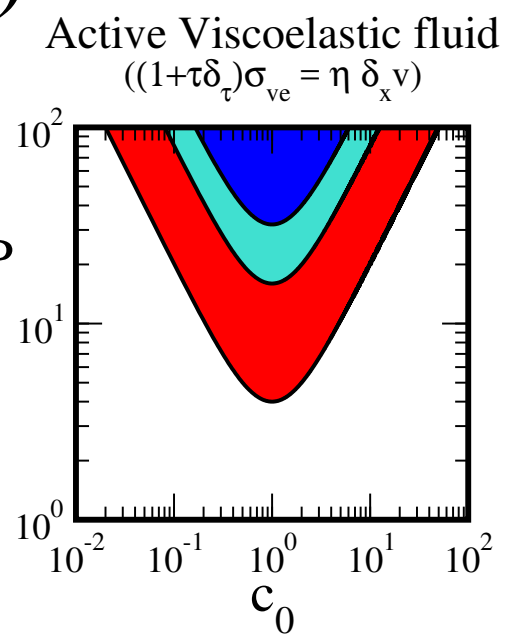

Figure 5. Phase diagrams of the models of active viscous fluid, active inviscid fluid with $\eta=0$ and viscoelastic fluid (Maxwell) of active matter. Phase diagrams (a-c) are obtained from linear stability analysis of the corresponding models keeping $E=10 \mathrm{kPa}$ for the viscoelastic model. White region corresponds to linearly stable homogeneous solutions, blue to unstable solutions with all eigenvalues positive and real, sky-blue to unstable solutions with all eigenvalues positive and complex, and red to real long wave instability. The color code is the same as for the different type of dispersion relations in Fig.4.

Table 3. Comparison of the resulting equations among viscoelastic models for living cells and examples from the bibliography where such approaches have been employed.

\begin{tabular}{lll}
\hline Viscoelastic model & Equations for the force balance & References \\
\hline Active & $\partial_{x} T-\gamma v=0$ & {$[41,50]$} \\
\hline Pure Viscous & $\eta \partial_{x}^{2} v+\partial_{x} T-\gamma v=0$ & {$[24,38]$} \\
\hline Pure elastic & $E \partial_{x}^{2} u+\partial_{x} T-\gamma v=0$ & {$[51,52,53]$} \\
\hline Maxwell & $\tau \gamma \partial_{t} v=\eta \partial_{x}^{2} v+\partial_{x} T-\gamma v+\tau \partial_{t} \partial_{x} T$ & {$[2,3,4,48,54,55,56]$} \\
\hline Kelvin-Voigt & $\eta \partial_{x}^{2} v+E \partial_{x}^{2} u+\partial_{x} T-\gamma v=0$ & {$[20,49,23,21,47,57,58,59,60]$} \\
\hline Standard & $\tau \gamma \partial_{t} v=E_{V} \partial_{x}^{2} u+\left(\eta+E_{V} \tau\right) \partial_{x}^{2} v+\partial_{x} T-\gamma v+\tau \partial_{t} \partial_{x} T$ & {$[34,45]$} \\
\hline Jeffrey & $\tau \gamma \partial_{t} v=\left(\eta_{v}+\eta_{m}\right) \partial_{x}^{2} v+\tau \eta_{v} \partial_{x}^{2} \partial_{t} v+\partial_{x} T-\gamma v+\tau \partial_{t} \partial_{x} T$ & {$[33]$} \\
\hline
\end{tabular}

formation of spatial patterns and introduce a simple isotropic active model for the polarization of cells [24]. The latter paper started with a simple active model with a purely viscous contribution $\sigma_{v e}=\eta \partial_{x} v$. The relation between the passive stress $\sigma_{v e}$ and the velocity of the fluid is:

$\partial_{x} \sigma_{v e}=\eta \partial_{x}^{2} v$

the corresponding equations of the model hence read:

$\partial_{t} c=-\partial_{x}(v c)+D \partial_{x}^{2} c$,

$0=\eta \partial_{x}^{2} v+\partial_{x} T-\gamma v$;

Linear stability analysis of the trivial homogeneous solution of Eqs.(9), i.e., $c=c_{0}$ and $v=0$ yields the following eigenvalue:

$\lambda=-D k^{2}\left(1-\frac{\frac{P}{P_{c}}}{1+\ell^{2} k^{2}}\right)$

where the characteristic hydrodynamic length is defined as $\ell^{2}=\eta / \gamma$, the Peclet number as $P=\xi /(D \gamma)$, and the critical Peclet number $P_{c}=\left(1+c_{0}\right)^{2} / c_{0}$. One can define also an additional dimensionless parameter $F=P / P_{c}$. For $F>1$ the solution is then unstable for a characteristic wavelength. If one checks the two eigenvalues corresponding to Eq.(7) the term $1+\ell^{2} k^{2}$ ensures negative eigenvalues for sufficiently large values of $k$. It means that in case of unstable eigenvalues there is always some stable modes and the system is stable to perturbations at small spatial scale. The corresponding instability curve is plotted in Fig.4(d) and corresponds to a long wave instability with real eigenmodes. The phase diagram is plotted in Fig.5(a). For $F>1$ the solution is unstable and all the modes plotted in Fig.5(a) are unstable giving rise to a long wave instability, see Fig.4(d). For $F<1$ the homogeneous solution is stable, see Fig.4(a). The parameter $P_{c}$ depends on $c_{0}$ and the corresponding value of $P$ needed to destabilize the homogeneous solution also changes, see Fig.5(a). For the special case $\eta=0, i$. $e$. for an active inviscid fluid, there is a competition between the advection produced by the active force, which controls the velocity of the 
fluid, see Eq.(1), and the diffusion, see both terms in Eq.(3). Such a competition has been used to study cytoplasmic streaming $[41,50]$ where the otherwise unphysical solutions are stabilized by the coupling to a second viscous phase.

If we apply such an approach to our active gel model we obtain the next set of equations:

$\partial_{t} c=-\partial_{x}(v c)+D \partial_{x}^{2} c$,

$0=\partial_{x} T-\gamma v$;

where the velocity depends only on the active tension. It has the same homogeneous solution as in the previous case. The linear stability analysis of such solution produces the eigenvalue:

$\lambda=-D k^{2}(1-F)$

For $F>1$ the solution is unstable and all the modes plotted in Fig.5(b) are unstable giving rise to an unphysical situation. For $F<1$ the homogeneous solution is stable. The phase diagram is the same as that the active fluid case discussed above, although the real long wave instability is converted to an unstable dispersion curve.

3.1.2. Maxwell model The Maxwell models has been employed in the derivation of the hydrodynamic equations of an active polar gel [61] where the flow velocity of the cytoskeleton is coupled with the asymmetric formation of a polar cytoskeleton, see for example [61, 2, 3, 4, 55]. Here we consider an isotropic nonpolar model, for which the Maxwell model is used in a viscoelastic isotropic active fluid to determine the viscoelastic properties and active stress responses in C. elegans and Zebrafish embryos [48]. The Maxwell model has been previously considered in the extension of a one-phase model to a two-phase model [54], where the cytoskeleton (active polar gel) and the cytosol (passive fluid) are described separately.

Different types of pattern formation are achieved by taking into consideration such passive viscoelastic response to active stresses. On the other hand, the response to external strains of an active viscoelastic model are similar to the passive Maxwell viscoelastic model [56].

The relation between the viscoelastic stress and the velocity of the fluid is in this case:

$\left(1+\tau \partial_{t}\right) \partial_{x} \sigma_{v e}=\eta \partial_{x}^{2} v$,

where $\tau=\eta / E$ is the viscoelastic relaxation time. The resulting equations of this viscoelastic model are:

$\partial_{t} c=-\partial_{x}(v c)+D \partial_{x}^{2} c$,

$\tau \gamma \partial_{t} v=\eta \partial_{x}^{2} v+\partial_{x} T-\gamma v+\tau \partial_{t} \partial_{x} T$

which yields the following characteristic equation for the eigenvalues:

$+\lambda^{2} \frac{\tau}{1+\ell^{2} k^{2}}$

$$
\begin{aligned}
& +\lambda\left(1+\frac{\tau}{1+\ell^{2} k^{2}} D k^{2}(1-F)\right) \\
& +D k^{2}\left(1-\frac{F}{1+\ell^{2} k^{2}}\right)=0
\end{aligned}
$$

This relation can give rise to three types of dispersion relations apart from the trivial stable case. As in the viscous active fluid it may produce a long wave instability with a characteristic wavelength, see Fig.4(d), or yield dispersion curves where the large wavenumber perturbation are all unstable as in Fig.4(b,c). The critical mechanochemical coupling strength, $\xi_{c}$ can be calculated analytically by considering the small $k$ limit of Eq. (12). We find

$\xi_{c}(E)=E \gamma P_{c}$,

which is equivalent to the condition $1-F=0$ and shows that the onset of mechanochemical instability is not dependent on the elastic modulus $E$. Just above onset the eigenvalues are real for all $k$, oscillatory eigenvalues appear for small $E$.

It is also instructive to look at the large $k$ limit of Eq. (12). Doing this, we can derive a condition where the dispersion curve undergoes a transition from a physically reasonable dispersion curve like in Fig. 4 (d) to a dispersion curve with diverging positive real parts as in Fig. 4 (c). It reads $\left(l^{2} / \tau+D(1-F)\right)^{2}<$ $(4 D l / \tau)$. The numerically computed phase diagram for a particular value of $E$ is shown in Fig.5(c) for comparison with more simple models of active fluid and confirms the analytical findings.

3.1.3. Kelvin-Voigt model The Kelvin-Voigt model has been employed for the elaboration of a onephase model of the cytoplasm considering the density changes of F-actin and the concentrations of myosin free and attached to the filaments of actin [49]. A two-component model consisting of an elastic network coupled frictionally to a permeating fluid is the basis of the two-phase model of the cytoplasm. Generic models of the solid actomyosin cytoskeleton and the fluid cytosol have been studied using a Kelvin-Voigt model for the passive viscoelasticity $[59,23]$. The modification of the strain-stress relation of the KelvinVoigt model under active cytoskeleton was considered in [59]. Such descriptions have been employed for the modeling of two cell types: modeling of muscle cells where the sarcomere chain can be described as a one dimensional elastic system in terms of a displacement field [59, 57] leading to oscillatory instabilities; and modeling of Physarum microplasmodia where the high concentration of actin produces elastic responses of the cytoplasm $[21,20]$.

The actin cytoskeleton of certain cells often condenses into stress fibers formed by filament bundles contracted by myosin motors. The Kelvin-Voigt model 
has been also utilized for the experimental study of the elastic properties of fiber bundles inside cells under stress to fit the elongation and the response of the cells after bundle incision [47]. Different models of active viscoelastic bundles were developed to explain traveling or standing filament density waves [60].

The viscoelastic stress in terms of the deformation and the velocity of the gel is:

$\partial_{x} \sigma_{v e}=E \partial_{x}^{2} u+\eta \partial_{x}^{2} v$,

where we note again that $v=\partial u / \partial t$. The equations of the model are:

$\partial_{t} c=-\partial_{x}(v c)+D \partial_{x}^{2} c$,

$0=\eta \partial_{x}^{2} v+E \partial_{x}^{2} u+\partial_{x} T-\gamma v$

$\partial_{t} u=v$

The corresponding characteristic equation for the eigenvalues describing perturbations of the homogeneous steady with $u=v=0$ and $c=c_{0}$ reads

$\lambda^{2}+\lambda D k^{2}\left(1-\frac{F-M}{1+\ell^{2} k^{2}}\right)+D^{2} k^{4} \frac{M}{1+\ell^{2} k^{2}}=0 ;$

where we have defined the nondimensional parameter $M=E /(D \gamma)$. The corresponding phase diagram is shown in Fig.6(a), indicating that the spatially uniform steady state undergoes an oscillatory long wave instability. The critical mechanochemical coupling strength, $\xi_{c}$ can be calculated analytically by considering the small $k$ limit. We find

$\xi_{c}(E)=(E+D \gamma) P_{c}$

which is equivalent to the condition $F-M=$ $E$ and shows that the critical coupling $\xi_{c}$ for the mechanochemical instability is linearly dependent on the elastic modulus $E$. The instability is also found to be of oscillatory nature with a frequency (expressed by the imaginary part of the critical eigenvalue) of $\omega=\left(D^{2} k^{4} M\right)^{1 / 2}$. If the large $k$ limit of Eq. (16) is considered, one finds that the eigenvalues for large wavenumbers $k$ stay always negative and the dispersion curve of the Kelvin-Voigt is hence always either of the type displayed in Fig. 4(e) or of the type shown in Fig. $4(\mathrm{f})$.

While for $M=0$ all eigenvalues are real, see Fig.4(d), and for large $M$ all the eigenvalues corresponding to unstable modes are complex, see Fig.4(f), for intermediate values of $M$ the most unstable mode is real, however there is still a range of wavenumbers with unstable complex eigenvalues, see Fig.4(e). The size of this range decreases with $M$, and disappears for $M=0$.

In the limit $\eta=0$ we obtain an active elastic solid model. The cytoskeleton network looks very stable and is elastic at short times. This allows for the construction of models of active elastic solids for the actomyosin network $[51,53]$ to evaluate the response

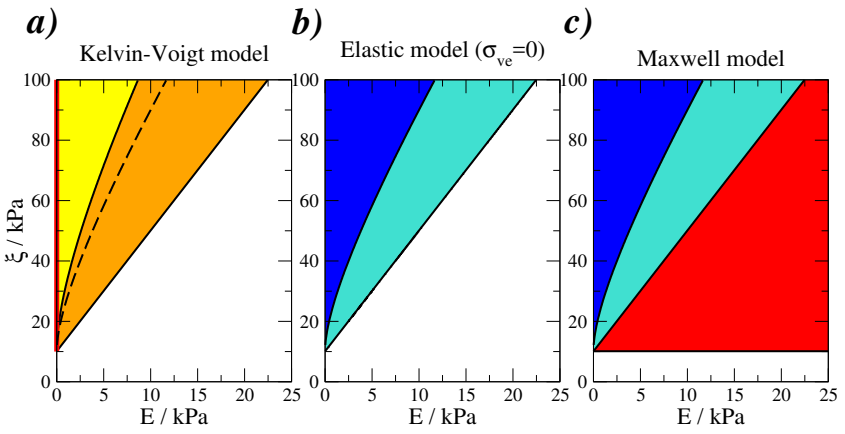

Figure 6. Phase diagrams of the elastic model keeping $\eta=0$ (a), the Kelvin-Voigt model keeping $\eta=25 \mathrm{kPa} \mathrm{s}(\mathrm{b})$ and the Maxwell model keeping $\eta=25 \mathrm{kPa} \mathrm{s}$ (c) for different values of $E$. White region corresponds to linearly stable homogeneous solutions, blue to unstable solutions with all eigenvalues positive and real, sky-blue to unstable solutions with all eigenvalues positive and complex, red to real long wave instability, and yellow to complex long wave instability and a complex long wave instability with real leading eigenmode. The color code is the same as for the different type of dispersion relations in Fig.4. Parameter values: $\ell=10 \mu \mathrm{m}, c_{0}=1$ and $D=10 \mu \mathrm{m}^{2} / \mathrm{s}$.

of the cell to external deformations. The acto-myosin elastic network is embedded in a permeating viscous fluid, with elastic response at long times and liquid-like dissipation at short times [52] giving rise to oscillations similar to the behavior of the full Kelvin-voigt model. The relation between the viscoelastic stress, and the deformation and the deformation of the gel is:

$\partial_{x} \sigma_{v e}=E \partial_{x}^{2} u$.

The equations of an elastic model of an active gel are:

$\partial_{t} c=-\partial_{x}(v c)+D \partial_{x}^{2} c$,

$0=E \partial_{x}^{2} u+\partial_{x} T-\gamma v$

$\partial_{t} u=v$

with the same homogeneous solution as in the previous cases. The eigenvalues fulfill the next condition from the linear stability analysis:

$\lambda^{2}+\lambda D k^{2}(1-(F-M))+D^{2} k^{4} M=0 ;$

The corresponding phase diagram of the instabilities obtained from the linear stability analysis is shown in Fig.6(b). The phase diagram is similar to the phase diagram for the non-zero value of $\eta$, but with unstable modes. The viscous term $\eta$ stabilizes the unstable modes, compare both diagrams Fig.6(a,b), by the introduction of the terms $1+\ell^{2} k^{2}$ in Eq.(16). Models with active elastic stress use permeating viscous fluids to account for the same effect $[53,52]$. Similarly, the active viscous fluid model stabilizes the modes of the active inviscid fluid model, see Fig5(a,b). 


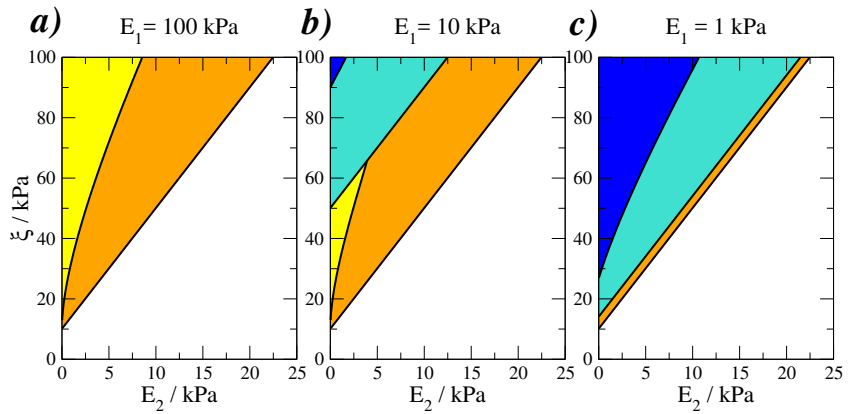

Figure 7. Phase diagram obtained from the linear stability analysis of the viscoelastic standard model of active matter for $E_{1}=1 \mathrm{kPa}(\mathrm{a}), E_{1}=10 \mathrm{kPa}(\mathrm{b})$ and $E_{1}=100 \mathrm{kPa}(\mathrm{c})$; keeping $\eta=25 \mathrm{kPa}$ s. Parameter values: $\ell=10 \mu \mathrm{m}, c_{0}=1$ and $D=10$ $\mu \mathrm{m}^{2} / \mathrm{s}$. The color code is the same as for the different type of dispersion relations in Fig. 4 .

\section{More complex viscoelastic active matter models}

4.0.1. Standard model The Kelvin-Voigt and the Maxwell models for viscoelasticity have only a small number of control parameters and are sometimes not suitable to fit experimental data. One can incorporate new dashpots and springs to the previous simple models to obtain more control parameters and different responses of the model for the cell material, see Fig.3. For the standard model, a spring in series with the dashpot is added to the equivalent circuit of the Kelvin-Voigt model. This model was employed to directly fit experiments with living cells in the measurement of viscoelastic properties by magnetic bead microrheometry [34] and other experimental techniques [45]. The relation between the viscoelastic stress and the deformation and velocity of the fluid is: $\left(1+\tau \partial_{t}\right) \partial_{x} \sigma_{v e}=\eta \partial_{x}^{2} v+E_{2} \tau \partial_{x}^{2} v+E_{2} \partial_{x}^{2} u$

where $\tau=\eta / E_{1}$ is the relaxation time associated to the first branch and $E_{2}$ is the elastic modulus associated to the isolated spring in the second branch.

The equations of the active model with this version of the viscoelasticity are:

$\partial_{t} c=-\partial_{x}(v c)+D \partial_{x}^{2} c$,

$\tau \gamma \partial_{t} v=E_{2} \partial_{x}^{2} u+\left(\eta+E_{2} \tau\right) \partial_{x}^{2} v+\partial_{x} T-\gamma v+\tau \partial_{t} \partial_{x} T$,

$\partial_{t} u=v$;

which produces the next characteristic equation for the corresponding eigenvalues:

$$
\begin{aligned}
& +\lambda^{3} \frac{\tau}{1+\ell^{2} k^{2}} \\
& +\lambda^{2}\left(1+\frac{\tau}{1+\ell^{2} k^{2}} D k^{2}(1-F+M)\right) \\
& +\lambda D k^{2}\left(1-\frac{F-M\left(1+D \tau k^{2}\right)}{1+\ell^{2} k^{2}}\right) \\
& +D^{2} k^{4} \frac{M}{1+\ell^{2} k^{2}}=0
\end{aligned}
$$

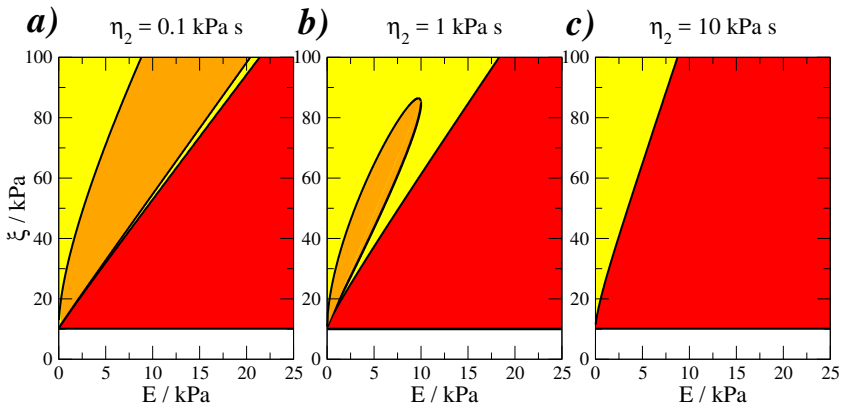

Figure 8. Phase diagram obtained from the linear stability analysis of the viscoelastic Jeffrey model of active matter for $\eta_{2}=0.1 \mathrm{kPa} \mathrm{s}(\mathrm{a}), \eta_{2}=1 \mathrm{kPa} \mathrm{s}(\mathrm{b})$ and $\eta_{2}=10 \mathrm{kPa} \mathrm{s}$ (c); keeping $\eta_{1}=25 \mathrm{kPa}$ s. Parameter values: $\ell=10 \mu \mathrm{m}, c_{0}=1$ and $D=10 \mu \mathrm{m}^{2} / \mathrm{s}$. The color code is the same as for the different type of dispersion relations in Fig.4.

where we define $M=E_{2} /(D \gamma)$ using the isolated spring. The critical mechanochemical coupling strength $\xi_{c}$ can be calculated analytically by considering the small $k$ limit. We recover the result obtained in Eq. (17) for the Kelvin-Voigt model above, which is equivalent to the condition $F-M=E$, i.e. the critical coupling $\xi_{c}$ for the mechanochemical instability is linearly dependent on the elastic modulus $E$. The instability is again found to be of oscillatory nature with a frequency (expressed by the imaginary part of the critical eigenvalue) of $\omega=\left(D^{2} k^{4} M\right)^{1 / 2}$. From the numerically determined phase diagram, see Fig.7, one finds, however, that the real part of the eigenvalues can diverge in the large $k$ limit at small values of $E_{2}$ for small choices of $E_{1}$ (the dispersion curves are then of the type displayed in Fig. 4(b,c) ). In the limit $\tau \rightarrow 0$ (keeping $\eta$ finite) one recovers Eq.(16) and therefore the Kelvin-Voigt limit. For $\tau \rightarrow \infty$ one simply obtains Eq.(20) corresponding to the purely elastic limit.

4.0.2. Jeffrey model The Jeffrey model of viscoelasticity incorporates an additional dashpot in parallel to the equivalent circuit used to derive the Maxwell model. It therefore represents a viscoelastic fluid in contrast to the standard model, which describes viscoelastic solid. This stress-strain relation of the Jeffrey model was employed for the study of rheology of two cell lines of fibroblasts subjected to a step stress to examine their mechanical response on different time scales [33].

The relation between the viscoelastic stress and the deformation and velocity of the fluid is for this case:

$\left(1+\tau \partial_{t}\right) \partial_{x} \sigma_{v e}=\eta \partial_{x}^{2} v+\eta_{2} \tau \partial_{x}^{2} \partial_{t} v$

where $\tau=\eta_{1} / E$ is the relaxation time of the first branch, associated to the dashpot with $\eta_{1}$. The resulting equations of the model are:

$\partial_{t} c=-\partial_{x}(v c)+D \partial_{x}^{2} c$ 
a)

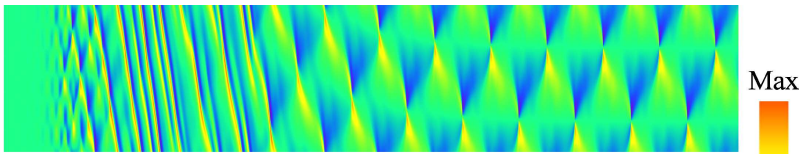

b)

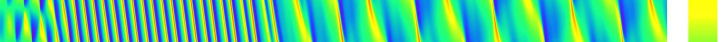
in 1.1

c)

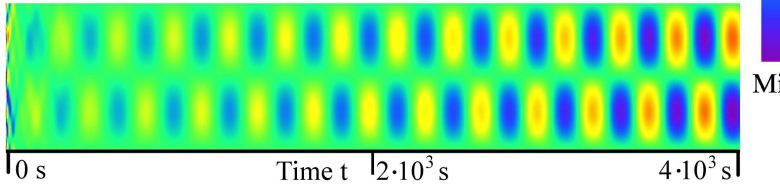

Figure 9. Space-time plot of the velocity field $v$ for the viscoelastic Standard model with $\tau=2.5 \mathrm{~s}\left(E_{1}=10 \mathrm{kPa}\right)$ and $E=7.5 k P a(\mathrm{a}), 8.25 \mathrm{kPa}$ (b), $12 \mathrm{kPa}$ (c).

$\tau \gamma \partial_{t} v=\eta \partial_{x}^{2} v+\tau \eta_{2} \partial_{x}^{2} \partial_{t} v+\partial_{x} T-\gamma v+\tau \partial_{t} \partial_{x} T$

where we have defined $\eta=\left(\eta_{1}+\eta_{2}\right)$. Linearization of this set of equations around the homogeneous steady state $v=0$ and $c=c_{0}$ gives the following characteristic equation for the eigenvalues $\lambda$ describing the temporal growth of modes with wavenumber $k$ :

$$
\begin{aligned}
& +\lambda^{2} \frac{\tau}{1+\ell^{2} k^{2}+\ell_{2}^{2} k^{2}}\left(1+\ell_{2}^{2} k^{2}\right) \\
& +\lambda\left(1+\frac{\tau}{1+\ell^{2} k^{2}+\ell_{2}^{2} k^{2}} D k^{2}\left(1-F+\ell_{2}^{2} k^{2}\right)\right) \\
& +D k^{2}\left(1-\frac{F}{1+\ell^{2} k^{2}+\ell_{2}^{2} k^{2}}\right)=0
\end{aligned}
$$

where $\ell_{2}^{2}=\eta_{2} / \gamma$ is a second hydrodynamic length scale and $\ell^{2}=\eta_{1} / \gamma$. For $\ell_{2}=0$ one recovers the Maxwell limit of Eq.(12), see in figure 8 the phase diagrams for different values of $\eta_{2}$. The critical mechanochemical coupling strength, $\xi_{c}$ of the Jeffrey model can as above be calculated analytically by considering the small $k$ limit. We find the same result as in Eq. (13), which is equivalent to the condition $1-F=0$ and shows that the onset of mechanochemical instability is not dependent on the elastic modulus $E$. Just above onset the eigenvalues are real for all $k$, oscillatory eigenvalues appear for small values of the elastic modulus $E$. The large $k$ limit yields that the eigenvalues there always have negative real parts.

\subsection{Numerical Simulations}

In order to obtain information about solutions beyond small perturbation around the steady state, we supplement the linear stability analysis by numerical simulations in one spatial dimension. For the details on the methods employed in the simulations see Appendix C.

4.1.1. Standard and Jeffrey models In this section we will only consider the Standard and the Jeffrey model.
Pattern formation in the Kelvin-Voigt model has previously been studied already in detail by numerical simulations in [19]. For both models under consideration here, we present a few representative cases for different parameter values that exhibit mechanochemical instabilities. As standard parameters we fix $\ell=10 \mu \mathrm{m}$, $c_{0}=1, D=10 \mu \mathrm{m}^{2} / \mathrm{s}, \eta=\eta_{1}=25 \mathrm{kPa} \mathrm{s}$, and $\xi=60 \mathrm{kPa}$, while the remaining values are varied. Periodic boundary conditions are used for all variables. For the initial regulator concentration a small random perturbation $\chi$ is added to the homogeneous distribution: $c(x, t=0)=c_{0}+\chi(x)$. The remaining quantities are set to zero.

Fig. 9 shows space-time plots for simulations of the viscoelastic Standard model with a constitutive law given by eq. (21). We limit the presentation of the spatiotemporal dynamics to one variable, the velocity $v$, since the other fields closely follow its dynamics and do not provide any novel (qualitative) information. In agreement with phase diagram in Fig. 7 (b), waves emerge by a (purely complex) instability. Close to the onset of instability (c) a small-amplitude standingwave pattern persists. Further from this onset $(a, b)$ there is a transient behavior until a stable wave pattern evolved. The transient pattern in particular includes a wave-coarsening process.

The results for the Jeffrey model (see eq. (24)) are presented in Fig. 10 for parameters belonging to the phase diagram in Fig. 8 (b). For an elastic modulus approaching to infinity the dynamics should converge to the purely viscous model. This is illustrated for a large value of $E$ in Fig. 10 (a) where the expected coarsening behavior is found. However, due to its finite value the remaining pulse travels slowly. When the parameters enter the region of linear complex eigenvalues the transient coarsening behavior changes, as shown in (b), by periodic modulations. However, the final state remains a traveling-pulse solution. For even lower elastic modulus $E$ multiple pulses may co-exist in a stable final state Fig. 10(c).

4.1.2. Nonlinear viscoelastic material Using linear viscoelastic constitutive laws as presented in the previous sections situations may occur, in which nontrivial velocity profiles remain static or move very slow. Consequently, deformations $f=1+\partial_{x} u$ grow in an unphysical manner or even become negative. To avoid this problem we introduce a nonlinear elastic constitutive law without changing the linear dispersion relations. A widely used hyperelastic model is the incompressible Mooney-Rivlin material. Since we consider one dimension we limit us to the uni-axial 
a)

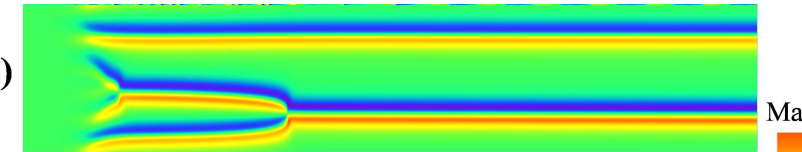

b)

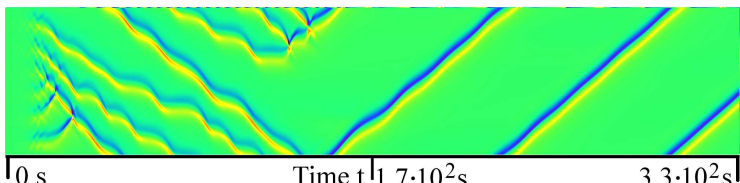

c)

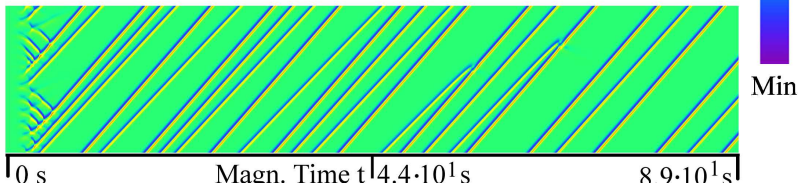

Figure 10. Space-time plot of the velocity field $v$ for the Jeffrey model with $\eta_{2}=1 \mathrm{kPas}$ and $E=25 \mathrm{kPa}(\tau=1 \mathrm{~s})$ (a), $E=7.75$ $\mathrm{kPa}(\tau=3.23 \mathrm{~s})(\mathrm{b})$ and $E=3 \mathrm{kPa}(\tau=8.33 \mathrm{~s})(\mathrm{c})$.

case with stress:

$\sigma_{M R}(f)=\left(C_{1}+C_{2} / f\right)\left(f-1 / f^{2}\right)$

$f=1+\partial_{x} u$.

From this one can conclude that the linear elastic modulus is $E=3\left(C_{1}+C_{2}\right)$. It is convenient to introduce $C_{1,2}=\frac{E}{6}(1 \pm \beta)$, where $\beta$ affects only the nonlinear response. For the viscoelastic Standard model we get:

$\left(1+\tau \partial_{t}\right) \sigma_{v e}=\left(\eta+\tau \sigma_{M R}^{\prime}\right) \partial_{x} v+\sigma_{M R}$.

However, for the active fluid models (Maxwell and Jeffrey) this modification is not applicable. An incompressibility constraint $\nabla \cdot \mathbf{v}=0$, where $\mathbf{v}$ is now a twoor three dimensional vector field is necessary in this case. In one dimension this constraint leads to trivial solutions. Therefore, we restrict the simulations to the Standard model.

In order to compare the dynamics between the linear and nonlinear case, in Fig. 11 (a,b) space-time plots with the same parameters as in Fig. 9 (a,b) are shown. The final states for the nonlinear material both show single traveling pulses. In sub-figure (c) the spatial profiles of this pulses are depicted. A direct comparison between the profiles of the linear and nonlinear constitutive law, see Fig. 11 (d) illustrates that the nonlinearity prevents deformations to become unphysical $(f \leq 0)$.

\section{Summary and discussion}

In this paper, we have investigated the influence of the passive material properties on the pattern formation properties of active viscoelastic fluids and solids. In general, sufficiently strong activity destabilizes the homogeneous steady states and leads to waves and patterns independent of the passive viscoelastic model a)

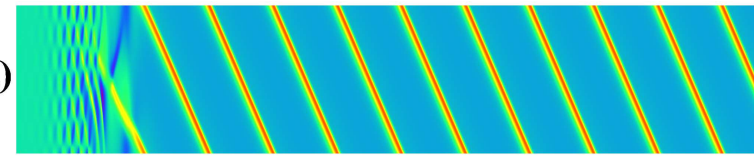

b)

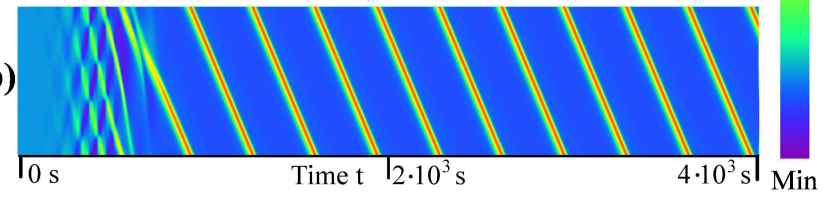

c)
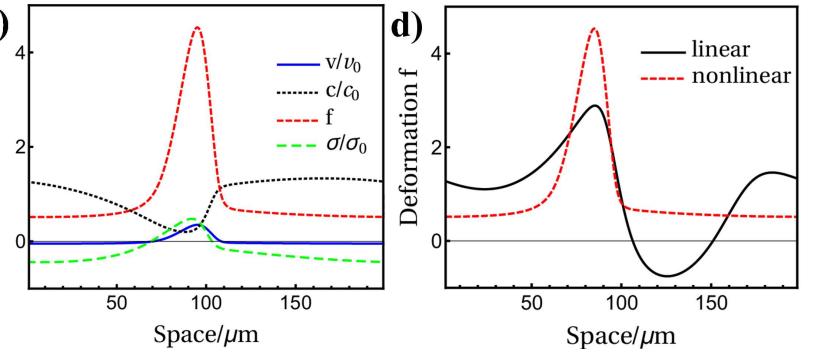

Figure 11. Space-time plot of the velocity field $v$ for the viscoelastic Standard model with nonlinear parallel spring element according to eq. (28). In (a) and (b) the same linear parameters where used as in Fig. 9 (a) and (b) and $\beta=0.5$. (c) Spatial profiles at a certain time for all relevant fields. The plotted quantities are normalized by $v_{0}=\frac{1}{\sqrt{\gamma \eta}} \frac{\xi}{P_{c}}, c_{0}$ and $\sigma_{0}=\xi / P_{c}$. (d) Comparison of the deformation profiles for the linear and nonlinear case corresponding to parameters in subfigure b).

chosen. The primary instability was found to be stationary for all active fluids considered, whereas all active solids exhibited on oscillatory primary instability. Since the concentration of the species regulating the active tension (i.e.concentration of molecular motors such as myosin or motor-regulating protein resp. motor-regulating ions such as calcium) , was assumed to be conserved in the system, all instabilities found are of long-wavelength nature. In a first step, we analysed the linear stability of the steady state with a spatially homogeneous concentration of motors or regulator species and zero fluid motion resp. time-independent deformation. Some of the models studied have the unphysical property of unstable eigenvalues in the large wave number limit, in particular the elastic solid model, the zero-viscosity limit of the simple active fluid model for all parameters as well as the Maxwell and Standard models for a substantial range of parameters. In contrast, the viscous active fluid model, the Jeffreys model for a viscoelastic fluids and the Kelvin-Voigt model for active solids have only negative eigenvalues in the large $k$ limit. This may be due to the fact that the isolated viscous dashpot added in parallel to elements with elastic springs in the equivalent circuits of Figure 2 provides a sufficiently large damping of perturbations for large wavenumbers in the full parameter range of the Kelvin-Voigt and Maxwell models. For two of 
models that have not been investigated before, namely the Jeffreys and standard models, we performed numerical simulations of the full nonlinear evolution equations. In general, the predictions of the linear stability were confirmed in these simulations.

In two-phase models of the cytoplasm suggested to model the dynamics of Physarum polycephalum the dynamics of the fluid viscous cytosol is taken into account in addition to the dynamics of the active solid viscoelastic cytoskeleton described by a Kelvin-Voigt model [20, 21, 23]. The resulting model equations are, however, apart from the sign of the active stress identical to the one-phase Kelvin-Voigt model given above and therefore, present, the best choice for modeling Physarum as an active solid.

Our study was restricted to one spatial dimension, but the instabilities found carry over also to the analogous two-dimensional equations as was already shown for the Kelvin-Voigt two-phase model of Physarum. Furthermore, only models of linear viscoelastic elasticity which are good approximations near the onset of instabilities and as long as deformations are small. We have obtained explicit expressions of the growth rates from the linear stability analysis as well as for the onset of instabilities. Some of the results were verified also in numerical simulations of the full nonlinear dynamics of two selected models. The springs and dashpots employed for the modeling of the viscoelastic properties do not correspond to any microscopic dynamics of molecules in living cells. They represent instead a phenomenological macroscopic approach. Other phenomenological models utilize computational tensegrity and are based in the description of prestresses of the interconnected filament structure, producing better descriptions for dynamical rheology of living cells [62]. Again another class of models start from the microscopic structure of the cytoskeleton $[1,29]$.

In summary, the motivation of this study was in part taken from results of the modeling the dynamics of the cytoplasm of Physarum, which employed a two-phase description coupling an active viscoelastic solid described by the Kelvin-Voigt model coupled to a simple passive, viscous liquid [19]. Here, specifically, we have performed a study of the influence of the viscoelastic properties of living cells by considering a number of simple models describing active solids and active fluids. In particular, we showed that all active fluid models exhibit a stationary long-wavelength instability with purely real eigenvalues, whereas all active solids models show an oscillatory long-wavelength instability involving complex eigenvalues with positive real parts. The threshold of the instability depends on the elastic modulus for all active solid models, while active fluid models display a constant threshold independent of the elastic modulus. For qualitative modelling of mechanochemical patterns, the main decision is clearly the one between active solids and active fluids. Among the considered solid models the Kelvin-Voigt model and among the fluid models studied the Jeffrey model were the best choices with respect to avoiding unstable modes with arbitrary large wavenumber in the whole parameter space. The Maxwell model and the standard model, in contrast, are only suitable to describe the behavior near the onset of the mechanochemical instability. Since all models studied are assuming linear elasticity, they should in any case not used in situations far away from onset where strong deformations occur. We demonstrated for an example in numerical simulations that in such situations nonlinear elasticity model are more suitable and will give results that are quite different from the ones found with linear elasticity. If experimental data of the viscoelastic response of the cell material that should be modelled are available, one may choose the model that gives the best fit to the data. The main finding reported above was that apart from the basic distinction if the cytoplasm responds as a fluid or a solid that the details of the material will not affect the potential for mechanochemical pattern formation. All cases are examples of mechano-chemical pattern formation [26, 27], i.e. they do not require chemical reaction-diffusion instabilities to structure the interior of a cell and the biochemical species regulating the active tension - either molecular motors or proteins resp. ions up- or downregulating the motor activity - are not undergoing chemical transformation or interactions. In typical biological situations, this picture is largely oversimplified. Instead often reaction-diffusion processes contribute substantially to formation of waves and patterns inside cells [63]. In other words, the simple mechanochemical models are usually coupled to reaction-diffusion equations, yielding patterns stemming from short-wave length Turing-type instabilities instead of the long-wavelength instabilities found for all models here. Bois et al. showed already that the coupling of reactiondiffusion equations to a mechanochemical model for an active fluid enhances the tendency of a system for pattern forming instabilities [24]. Alonso et al. observed a similar trend for the coupling of the active poroelastic equations to reaction-diffusion equations [21]. Radszuweit et al. [20] showed that the whole variety of deformations pattern observed in experiments with plasmodial droplets of Physarum polycephalum reported in $[8,9,10,11]$ require also an extension of the active poroelastic model by reactiondiffusion equations describing the regulation of calcium ions. 
Table A1. List of parameters employed along the manuscript with their meaning.

\begin{tabular}{lll}
\hline Parameter & Expression & Meaning \\
\hline$\sigma$ & & total stress in the continuous \\
$\sigma_{v e}$ & & viscoelastic stress \\
$T$ & & Active tension by motors \\
$C$ & & Concentration of the regulator \\
$K_{c}$ & $C / K_{c}$ & Characteristic concentration \\
$c$ & & Dimensionless concentration \\
$c_{o}$ & & Diffusion coefficient of $c$ \\
$D$ & & Coupling regulator-tension \\
$\xi$ & & Local velocity of the gel \\
$v$ & & Local deformation of the gel \\
$u$ & & Friction coefficient \\
$\gamma$ & & Viscosity of the gel \\
$\eta$ & $\xi$ & Peclet number \\
$P$ & $\left(1+c_{0}\right)^{2} / c_{0}$ & Critical Peclet number \\
$P_{c}$ & $\sqrt{\eta / \gamma}$ & Characteristic length \\
$\ell$ & $P / P_{c}$ & Non-dimensional Peclet number \\
$F$ & $\eta / E$ & elastic modulus of the gel \\
$E$ & $E / D \gamma$ & characteristic relaxation time \\
$\tau$ & & Non-dimensional elastic modulus \\
$M$ & &
\end{tabular}

\section{Acknowledgments}

We acknowledge financial support from MINECO of Spain (SA) under project FIS-2014-55365-P and the Ramon y Cajal program with the grant number RYC-2012-11265; from the German Science Foundation (DFG) within the framework of GRK 1558 (MR,HE,MB) Nonequilibrium Collective Dynamics in Condensed Matter and Biological Systems and SFB910 Control of self-organizing nonlinear systems.

\section{Appendix A. List and meaning of parameters}

An complete list with the parameters employed in the manuscript is shown in tab.A1, together with a short explanation of the meaning of the parameter and the analytical expression if is necessary.

\section{Appendix B. Strain-stress relations}

Next we list the relation between the applied stress and the corresponding strain obtained from the spring and dashpots models shown in Fig.2 and used for the calculations shown in Fig.3. Note that the total stress in two parallel elements is the sum of both stresses; and the strain of two elements in serie is the sum of both strains.

- The elastic model is based in a linear relation between the stress and the strain $\sigma=E \epsilon$ and therefore:

$$
\frac{d \epsilon(t)}{d t}=\frac{1}{E} \frac{d \sigma(t)}{d t}
$$

- The viscous model is

$$
\frac{d \epsilon(t)}{d t}=\frac{\sigma(t)}{\eta}
$$

- The Kelvin-Voigt model is

$$
\frac{d \epsilon(t)}{d t}=\frac{\sigma(t)}{\eta}-\frac{E}{\eta} \epsilon(t)
$$

- The Maxwell model is

$$
\frac{d \epsilon(t)}{d t}=\frac{\sigma(t)}{\eta}+\frac{1}{E} \frac{d \sigma(t)}{d t}
$$

- The Standard model, using $E=E_{1}+E_{2}$, is

$$
\frac{d \epsilon(t)}{d t}=\frac{1}{E} \frac{d \sigma(t)}{d t}+\frac{E_{2}}{\eta E} \sigma(t)-\frac{E_{1} E_{2}}{\eta E} \epsilon(t)
$$

- The Jeffrey model, using $\eta=\eta_{1}+\eta_{2}$, is

$$
\frac{\eta_{1} \eta_{2}}{\eta E} \frac{d^{2} \epsilon(t)}{d t^{2}}+\frac{d \epsilon(t)}{d t}=\frac{\sigma(t)}{\eta}+\frac{\eta_{2}}{\eta E} \frac{d \sigma(t)}{d t}
$$

\section{Appendix C. Numerical methods}

A staggered Finite Volume and Finite Difference scheme for discretization in space is used. If $n$ is the spatial index, then concentration values $c_{n}$ are located at position $x_{n}$, while velocity $v_{n+\frac{1}{2}}$, stress $\sigma_{n+\frac{1}{2}}$ and displacement $u_{n+\frac{1}{2}}$ are located at $x_{n+\frac{1}{2}}=x_{n}+\Delta x / 2$ on an equidistant grid. For interpolation of the advective flux $j=v c$ at nodes $n+\frac{1}{2}$ the upwind scheme is applied. For time discretization of eq. (3) and the stress-strain relation we chose the implicit Euler scheme to improve numerical stability. By posterior check of the simulation results we ensure that the associated numerical diffusion coefficient $D_{n u m} \approx v \Delta x / 2$ is much smaller than the regulator diffusion $D$. In the presented cases we set the domain length to $L=200 \mu \mathrm{m}$ and the the space step to $\Delta x=0.1 \mu \mathrm{m}$. The time-step size $\Delta t$ varies from $0.25 \mathrm{~s}-0.4 \mathrm{~s}$ since it influences numerical stability. In order to solve the nonlinear system occurring in each time step we make use of a staggered equation scheme and iterate until convergence within some tolerance. The software package UMFPACK [64] provides us with a reliable solver for systems of linear equations.

[1] F. Huber, J. Schnauß, S. Rönicke, P. Rauch, K. Müller, C. Fütterer, and J. Käs, "Emergent complexity of the cytoskeleton: from single filaments to tissue," Advances in physics, vol. 62, no. 1, pp. 1-112, 2013.

[2] J.-F. Joanny and J. Prost, "Active gels as a description of the actin-myosin cytoskeleton," HFSP journal, vol. 3, no. 2, pp. 94-104, 2009.

[3] M. Marchetti, J. Joanny, S. Ramaswamy, T. Liverpool, J. Prost, M. Rao, and R. A. Simha, "Hydrodynamics of soft active matter," Reviews of Modern Physics, vol. 85, no. 3, p. 1143, 2013.

[4] J. Prost, F. Jülicher, and J. Joanny, "Active gel physics," Nature Physics, vol. 11, no. 2, pp. 111-117, 2015.

[5] M. R. Mofrad and R. D. Kamm, Cytoskeletal mechanics: models and measurements in cell mechanics. Cambridge University Press, 2006. 
[6] G. Isenberg and K. Wohlfarth-Bottermann, "Transformation of cytoplasmic actin importance for the organization of the contractile gel reticulnm and the contractionrelaxation cycle of cytoplasmic actomyosin," Cell and tissue research, vol. 173, no. 4, pp. 495-528, 1976.

[7] W. Stockem and K. Brix, "Analysis of microfilament organization and contractile activities in physarum," International review of cytology, vol. 149, pp. 145-215, 1994.

[8] S. Takagi and T. Ueda, "Emergence and transitions of dynamic patterns of thickness oscillation of the plasmodium of the true slime mold physarum polycephalum," Physica D: Nonlinear Phenomena, vol. 237, no. 3, pp. 420-427, 2008.

[9] S. Yoshiyama, M. Ishigami, A. Nakamura, and K. Kohama, "Calcium wave for cytoplasmic streaming of physarum polycephalum," Cell biology international, vol. 34, no. 1, pp. 35-40, 2010.

[10] E. Bernitt, C. Oettmeier, and H.-G. Döbereiner, "Microplasmodium dynamics of physarum polycephalum," in 6th World Congress of Biomechanics (WCB 2010). August 1-6, 2010 Singapore, pp. 1133-1136, Springer, 2010 .

[11] S. Tsuda and J. Jones, "The emergence of synchronization behavior in physarum polycephalum and its particle approximation," Biosystems, vol. 103, no. 3, pp. 331$341,2011$.

[12] A. Fessel, C. Oettmeier, E. Bernitt, N. C. Gauthier, and H.-G. Döbereiner, "Physarum polycephalum percolation as a paradigm for topological phase transitions in transportation networks," Physical review letters, vol. 109, no. 7, p. 078103, 2012.

[13] W. Baumgarten and M. J. Hauser, "Dynamics of frontal extension of an amoeboid cell," EPL (Europhysics Letters), vol. 108, no. 5, p. 50010, 2014.

[14] O. L. Lewis, S. Zhang, R. D. Guy, and J. C. del Álamo, "Coordination of contractility, adhesion and flow in migrating physarum amoebae," Journal of The Royal Society Interface, vol. 12, no. 106, p. 20141359, 2015.

[15] J.-P. Rieu, H. Delanoë-Ayari, S. Takagi, Y. Tanaka, and T. Nakagaki, "Periodic traction in migrating large amoeba of physarum polycephalum," Journal of the Royal Society Interface, vol. 12, no. 106, p. 20150099, 2015.

[16] B. Rodiek, S. Takagi, T. Ueda, and M. J. Hauser, "Patterns of cell thickness oscillations during directional migration of physarum polycephalum," European Biophysics Journal, vol. 44, no. 5, pp. 349-358, 2015.

[17] S. Zhang, R. D. Guy, J. C. Lasheras, and J. C. del Alamo, "Self-organized mechano-chemical dynamics in amoeboid locomotion of physarum fragments," Journal of Physics D: Applied Physics, vol. 50, no. 20, p. 204004, 2017.

[18] T. Nakagaki and R. D. Guy, "Intelligent behaviors of amoeboid movement based on complex dynamics of soft matter," Soft Matter, vol. 4, no. 1, pp. 57-67, 2008.

[19] M. Radszuweit, H. Engel, and M. Bär, "A model for oscillations and pattern formation in protoplasmic droplets of physarum polycephalum," The European Physical Journal Special Topics, vol. 191, no. 1, pp. 159$172,2010$.

[20] M. Radszuweit, H. Engel, and M. Bär, "An active poroelastic model for mechanochemical patterns in protoplasmic droplets of physarum polycephalum," PLOS one, vol. 9, p. e99220, 2014.

[21] S. Alonso, U. Strachauer, M. Radszuweit, M. Bär, and M. J. Hauser, "Oscillations and uniaxial mechanochemical waves in a model of an active poroelastic medium: Application to deformation patterns in protoplasmic droplets of physarum polycephalum," Physica D: Nonlinear Phenomena, vol. 318-319, pp. 58-69, 2016.
[22] V. A. Teplov, "Role of mechanics in the appearance of oscillatory instability and standing waves of the mechanochemical activity in the physarum polycephalum plasmodium," Journal of Physics D: Applied Physics, vol. 50, no. 21, p. 213002, 2017.

[23] M. Radszuweit, S. Alonso, H. Engel, and M. Bär, "Intracellular mechanochemical waves in an active poroelastic model," Physical review letters, vol. 110, no. 13, p. $138102,2013$.

[24] J. S. Bois, F. Jülicher, and S. W. Grill, "Pattern formation in active fluids," Physical review letters, vol. 106, no. 2, p. $028103,2011$.

[25] M. Mayer, M. Depken, J. S. Bois, F. Jülicher, and S. W. Grill, "Anisotropies in cortical tension reveal the physical basis of polarizing cortical flows," Nature, vol. 467, no. 7315, pp. 617-621, 2010.

[26] J. Howard, S. W. Grill, and J. S. Bois, "Turing's next steps: the mechanochemical basis of morphogenesis," Nature Reviews Molecular Cell Biology, vol. 12, no. 6, pp. 392398, 2011.

[27] P. Gross, K. V. Kumar, and S. W. Grill, "How active mechanics and regulatory biochemistry combine to form patterns in development," Annual Review of Biophysics, vol. 46, no. 1, pp. 337-356, 2017.

[28] Y. Zhang, H. Kawamichi, H. Tanaka, S. Yoshiyama, K. Kohama, and A. Nakamura, "Calcium-dependent regulation of the motor activity of recombinant fulllength physarum myosin," Journal of biochemistry, vol. 152 , no. 2, pp. 185-190, 2012.

[29] P. Kollmannsberger and B. Fabry, "Linear and nonlinear rheology of living cells," Annual Review of Materials Research, vol. 41, pp. 75-97, 2011.

[30] P. A. Pullarkat, P. A. Fernández, and A. Ott, "Rheological properties of the eukaryotic cell cytoskeleton," Physics Reports, vol. 449, no. 1, pp. 29-53, 2007.

[31] X. Trepat, G. Lenormand, and J. J. Fredberg, "Universality in cell mechanics," Soft Matter, vol. 4, no. 9, pp. 17501759, 2008.

[32] S.-Y. Tee, A. R. Bausch, and P. A. Janmey, "The mechanical cell," Current Biology, vol. 19, no. 17, pp. R745-R748, 2009.

[33] F. Wottawah, S. Schinkinger, B. Lincoln, R. Ananthakrishnan, M. Romeyke, J. Guck, and J. Käs, "Optical rheology of biological cells," Physical review letters, vol. 94, no. 9, p. 098103, 2005.

[34] A. R. Bausch, F. Ziemann, A. A. Boulbitch, K. Jacobson, and E. Sackmann, "Local measurements of viscoelastic parameters of adherent cell surfaces by magnetic bead microrheometry," Biophysical Journal, vol. 75, no. 4, pp. 2038-2049, 1998.

[35] M. Kapustina, G. E. Weinreb, N. Costigliola, Z. Rajfur, K. Jacobson, and T. C. Elston, "Mechanical and biochemical modeling of cortical oscillations in spreading cells," Biophysical journal, vol. 94, no. 12, pp. 4605-4620, 2008.

[36] N. Costigliola, M. T. Kapustina, G. E. Weinreb, A. Monteith, Z. Rajfur, T. C. Elston, and K. Jacobson, "Rhoa regulates calcium-independent periodic contractions of the cell cortex," Biophysical journal, vol. 99, no. 4, pp. 1053-1063, 2010.

[37] E. Hannezo, B. Dong, P. Recho, J.-F. Joanny, and S. Hayashi, "Cortical instability drives periodic supracellular actin pattern formation in epithelial tubes," Proceedings of the National Academy of Sciences, vol. 112, no. 28, pp. 8620-8625, 2015.

[38] K. V. Kumar, J. S. Bois, F. Jülicher, and S. W. Grill, "Pulsatory patterns in active fluids," Physical Review Letters, vol. 112, no. 20, p. 208101, 2014.

[39] K. Kohama, "Calcium inhibition as an intracellular signal for actin-myosin interaction," Proceedings of the Japan 
Academy, Series B, vol. 92, no. 10, pp. 478-498, 2016.

[40] K. Luby-Phelps, "Cytoarchitecture and physical properties of cytoplasm: volume, viscosity, diffusion, intracellular surface area," International review of cytology, vol. 192, pp. 189-221, 1999.

[41] R. E. Goldstein and J.-W. van de Meent, "A physical perspective on cytoplasmic streaming," Interface Focus, vol. 5, no. 4, p. 20150030, 2015.

[42] H. T. Banks, S. Hu, and Z. R. Kenz, "A brief review of elasticity and viscoelasticity for solids," Advances in Applied Mathematics and Mechanics, vol. 3, no. 01, pp. 1-51, 2011.

[43] T. Betz, D. Koch, Y.-B. Lu, K. Franze, and J. A. Käs, "Growth cones as soft and weak force generators," Proceedings of the National Academy of Sciences, vol. 108, no. 33, pp. 13420-13425, 2011.

[44] M. Radmacher, M. Fritz, C. M. Kacher, J. P. Cleveland, and P. K. Hansma, "Measuring the viscoelastic properties of human platelets with the atomic force microscope," Biophysical journal, vol. 70, no. 1, pp. 556-567, 1996.

[45] O. Thoumine and A. Ott, "Time scale dependent viscoelastic and contractile regimes in fibroblasts probed by microplate manipulation," Journal of cell science, vol. 110, no. 17, pp. 2109-2116, 1997.

[46] S. Ganguly, L. S. Williams, I. M. Palacios, and R. E. Goldstein, "Cytoplasmic streaming in drosophila oocytes varies with kinesin activity and correlates with the microtubule cytoskeleton architecture," Proceedings of the National Academy of Sciences, vol. 109, no. 38, pp. 15109-15114, 2012 .

[47] S. Kumar, I. Z. Maxwell, A. Heisterkamp, T. R. Polte, T. P. Lele, M. Salanga, E. Mazur, and D. E. Ingber, "Viscoelastic retraction of single living stress fibers and its impact on cell shape, cytoskeletal organization, and extracellular matrix mechanics," Biophysical journal, vol. 90, no. 10, pp. 3762-3773, 2006.

[48] A. Saha, M. Nishikawa, M. Behrndt, C.-P. Heisenberg, F. Jülicher, and S. Grill, "Determining physical properties of the cell cortex," Biophysical journal, vol. 110 , no. 6 , pp. 1421-1429, 2016.

[49] K. Larripa and A. Mogilner, "Transport of a 1d viscoelastic actin-myosin strip of gel as a model of a crawling cell," Physica A: Statistical Mechanics and its Applications, vol. 372 , no. 1, pp. 113-123, 2006.

[50] P. K. Trong, J. Guck, and R. E. Goldstein, "Coupling of active motion and advection shapes intracellular cargo transport," Physical review letters, vol. 109, no. 2, p. 028104, 2012.

[51] F. C. MacKintosh and A. J. Levine, "Nonequilibrium mechanics and dynamics of motor-activated gels," Physical review letters, vol. 100, no. 1, p. 018104, 2008.

[52] S. Banerjee, T. B. Liverpool, and M. C. Marchetti, "Generic phases of cross-linked active gels: relaxation, oscillation and contractility," EPL (Europhysics Letters), vol. 96, no. 5 , p. $58004,2011$.

[53] R. J. Hawkins and T. B. Liverpool, "Stress reorganization and response in active solids," Physical review letters, vol. 113, no. 2, p. 028102, 2014.

[54] A. Callan-Jones and F. Jülicher, "Hydrodynamics of active permeating gels," New Journal of Physics, vol. 13, no. 9, p. 093027, 2011.

[55] F. Juelicher, K. Kruse, J. Prost, and J.-F. Joanny, "Active behavior of the cytoskeleton," Physics Reports, vol. 449, no. 1 , pp. 3-28, 2007.

[56] J. J. Muñoz and S. Albo, "Physiology-based model of cell viscoelasticity," Physical Review E, vol. 88, no. 1, p. $012708,2013$.

[57] S. Günther and K. Kruse, "Spontaneous waves in muscle fibres," New Journal of Physics, vol. 9, no. 11, p. 417, 2007.
[58] A. Besser, J. Colombelli, E. H. Stelzer, and U. S. Schwarz, "Viscoelastic response of contractile filament bundles," Physical Review E, vol. 83, no. 5, p. 051902, 2011.

[59] S. Banerjee and M. C. Marchetti, "Instabilities and oscillations in isotropic active gels," Soft Matter, vol. 7, no. 2, pp. 463-473, 2011.

[60] R. Peter, V. Schaller, F. Ziebert, and W. Zimmermann, "Pattern formation in active cytoskeletal networks," New Journal of Physics, vol. 10, no. 3, p. 035002, 2008.

[61] K. Kruse, J.-F. Joanny, F. Jülicher, J. Prost, and K. Sekimoto, "Generic theory of active polar gels: a paradigm for cytoskeletal dynamics," The European Physical Journal E, vol. 16, no. 1, pp. 5-16, 2005.

[62] D. E. Ingber, N. Wang, and D. Stamenović, "Tensegrity, cellular biophysics, and the mechanics of living systems," Reports on Progress in Physics, vol. 77, no. 4, p. 046603, 2014.

[63] C. Beta and K. Kruse, "Intracellular oscillations and waves," Annual Review of Condensed Matter Physics, vol. 8, pp. 239-264, 2017.

[64] T. A. Davis, "Algorithm 832: Umfpack v4. 3an unsymmetric-pattern multifrontal method," $A C M$ Transactions on Mathematical Software (TOMS), vol. 30, no. 2, pp. 196-199, 2004. 